\title{
Biomarqueurs de toxicité et anomalies métaboliques dans les principales intoxications graves. Symptomatologie clinique et toxique. Le prélèvement conservatoire
}

\section{Laboratory guidelines for the investigation of patient poisoning, clinical signs, and biological sample storage}

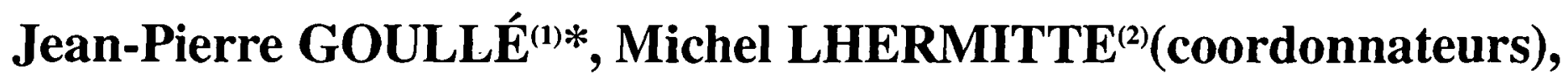
Mireille BARTHOLI ${ }^{(3)}$, Jean-Christophe BOYER ${ }^{(4)}$, Bernard CAPOLAGHI(s), Corinne CHARLIER ${ }^{(6)}$, Vincent DANEL ${ }^{(7)}$, Gérard DESCH ${ }^{(8)}$, Alain FEUILLU ${ }^{(9)}$, Bernard FLOUVAT ${ }^{(10)}$, Daniel MATHIEU ${ }^{(11)}$, Patrick NISSE ${ }^{(12)}$, Nourédine SADEG ${ }^{(13)}$, Anton SZYMANOWICZ ${ }^{(1)}$ (Groupe de travail pluridisciplinaire SFBC-SFTA-STC Toxicologie et Biologie Clinique)

(1) Laboratoire de Pharmacocinétique et de Toxicologie Cliniques, GH, LE HAVRE (2) Laboratoire de Biochimie et de Biologie Moléculaire, CHRU, LILLE

(3) Laboratoire de Biochimie, CH, CHAMBÉRY

(4) Laboratoire de Biochimie, CHRU, NÎMES

(5) Laboratoire de Biologie, CHR, THIONVILLE

(6) Laboratoire de Toxicologie Clinique, CHU, LIÈGE

(7) Unité de Toxicologie Clinique, CHRU, GRENOBLE (8) Laboratoire de Biochimie-Toxicologie, CH, AVIGNON

(9) Laboratoire des Services de Réanimation, CHRU, RENNES (10) Laboratoire de Toxicologie-Pharmacologie, Hôpital Ambroise Paré, BOULOGNE BILLANCOURT

(11) Service de Réanimation Médicale, CHRU, LILLE

(12) Centre Antipoison, CHRU, LILLE

(13) Laboratoire, $\mathrm{CH}$, PONTOISE (14) Laboratoire de Biochimie, CH ROANNE

* Tirés à part : Jean-Pierre GOULLÉ, Laboratoire de Pharmacocinétique et de Toxicologie.Cliniques, Groupe Hospitalier du Havre - BP 24 - 76083 LE HAVRE Cedex - FRANCE Tél : 0232733223 - Fax : 0232733238 -E-mail jgoulle@ch-havre.fr Article publié dans les Annales de Biologie Clinique, 2003 ; 61 : 421-33 et reproduit avec l'aimable autorisation de l'éditeur. 


\section{RÉSUMÉ}

Les membres du groupe de travail pluridisciplinaire "Toxicologie et Biologie Clinique» appartenant à la Société Française de Biologie Clinique (SFBC), à la Société Française de Toxicologie Analytique (SFTA), à la Société de Toxicologie Clinique (STC), proposent une aide méthodologique à l'intention des biologistes non spécialistes en toxicologie. Ils formulent également pour respecter les bonnes pratiques de laboratoire, un certain nombre de recommandations. Trois documents de synthèse ont été élaborés. Ils concernent d'une part, les biomarqueurs de toxicité et les anomalies métaboliques dans les principales intoxications graves, d'autre part les symptômes cliniques essentiels correspondant à ces intoxications, enfin des recommandations sur le prélèvement conservatoire. Pour les biomarqueurs de toxicité, le tableau de synthèse concerne une cinquantaine de xénobiotiques : les principaux symptômes, les méthodes d'identification ou de dosage disponibles en urgence, les marqueurs biologiques utiles, la décision clinique correspondante, les dosages éventuels nécessaires à la prise en charge du patient, ainsi que l'antidote lorsque celui-ci existe. En ce qui concerne les techniques, le groupe propose un certain nombre de recommandations relatives au domaine clinique et médico-légal. Le groupe insiste également sur la nécessité de procéder dans certains cas à des prélèvements biologiques conservatoires dès la prise en charge du malade, prélèvements qui seront ou ne seront pas analysés en fonction du contexte et/ou de l'évolution de l'intoxication.

\section{MOTS-CLÉS}

Biologie Clinique, Toxicologie, Guide Méthodologique

\section{Introduction}

Au cours des dix dernières années, la toxicologie clinique a fortement évolué. Les progrès analytiques ont été considérables. Les causes d'intoxications se sont diversifiées et les médicaments occupent aujourd'hui la première place (1-3). Quant à l'évolution des ordonnances médicales, les benzodiazépines ont remplacé les barbituriques et le paracétamol est maintenant beaucoup plus prescrit que l'aspirine. On assiste également à une nette progression de l'utilisation des produits stupéfiants, le cannabis en particulier chez les adolescents (4). L'émergence de pathologies nouvelles liées à l'usage de cannabis chez des sujets jeunes constitue aussi un problème majeur de santé publique (5). En ce qui concerne les progrès dans le domaine de l'analyse, ceux-ci sont tels qu'à l'heure actuelle pratiquement tous les toxiques peuvent être identifiés et voire quantifiés dans les milieux biologiques. Le détecteur de masse s'impose en tant que détecteur universel, qu'il soit couplé à un chromatographe en phase gazeuse (CPG-SM), ou en phase liquide (CL-SM), ou associé à un plasma à couplage inductif (ICP-SM). Pour faire la synthèse de nos connaissances dans le domaine de l'analyse toxicologique, le comité scientifique de la SFBC (Société Française de Biologie Clinique,

\section{SUMMARY}

The members of the joint group "Toxicology and Clinical Biology» of the French Society of Clinical Biology (SFBC), the French Society of Analytical Toxicology (SFTA), and the Society of Clinical Toxicology (STC), suggest guidelines to meet the requirements of clinical biologists who are not specialized in toxicology. In order to respect good laboratory practices, they propose a certain number of guidelines. Three synthesis tables have been established. They are, on one hand, about toxicity biomarkers and metabolic disorders associated with the main severe intoxications, on the other hand about clinical signs that are observed during these intoxications, and finally about precautionary arrangements for biological sampling. The table also relates to approximately fifty xenobiotics : main clinical signs, emergency identification or quantification of the suspected product, useful biological markers, therapeutic decision, necessary quantitations to take patient care into consideration, as well as poison antidotes, are described. Recommendations regarding medical and forensic techniques are also proposed by the group. It is also necessary to collect and store biological samples as soon as the patients are taken care of. These samples will be analyzed or not, depending on the individual case history.

\section{KEY-WORDS}

Clinical Biology, Toxicology, Guidelines.

Président : Pr F. TRIVIN) a constitué un groupe de travail qui associe la Société Française de Toxicologie Analytique (SFTA), société savante dans le domaine (Président : Dr P. KINTZ) et la Société de Toxicologie Clinique (STC), maillon indispensable à la prise en charge globale du malade (Président : Dr V. DANEL).

Le présent rapport est destiné à apporter une aide méthodologique par des spécialistes de toxicologie analytique et clinique à des biologistes généralistes.

\section{Toxicologie et biologie cli- nique}

Il convient de rappeler que la prise en charge d'une intoxication ne se limite pas à l'analyse toxicologique et que le bilan biologique peut être aussi important, sinon plus, que l'analyse toxicologique. Quelques exemples le démontrent :

- dans une intoxication par anticoagulant, la recherche et le dosage de l'anticoagulant ne sont pas nécessaires à la prise en charge du malade et à la mise en œuvre d'un traitement, alors que le taux de prothrombine (TP) et le rapport normalisé international (INR) sont des marqueurs biologiques indispensables. 
- en cas d'intoxication par cyanures (incendie, ingestion de cyanure), les lactates et les gaz du sang sont deux examens biologiques essentiels. La lactatémie > $10 \mathrm{mmol} / \mathrm{l}$ constitue un signe de gravité, alors que le dosage des cyanures, même s'il est utile, n'est pas nécessaire à la prise en charge du patient, ni à l'instauration d'un traitement antidotique précoce.

- dans une hypoglycémie secondaire à une ingestion de médicament hypoglycémiant, si l'identification de l'agent causal est indispensable pour le diagnostic, $\mathrm{pH}$, lactates, glycémie et trou anionique doivent être réalisés en première intention. Ces éléments sont suffisants pour perfuser le malade sans délai.

Pour aider les biologistes généralistes, non spécialistes de toxicologie, le groupe a élaboré deux documents de synthèse :

- biomarqueurs de toxicité et anomalies métaboliques dans les principales intoxications graves (Annexe 1). Pour les biomarqueurs de toxicité d'une cinquantaine de xénobiotiques, le tableau aborde successivement les principaux symptômes, les méthodes d'identification ou de dosage disponibles en urgence, les marqueurs biologiques utiles, l'attitude thérapeutique, les dosages éventuels nécessaires à la prise en charge du patient -y compris parfois les dosages toxicocinétiques-, ainsi que l'antidote lorsque celui-ci existe.

- approche clinique de ces principales intoxications graves avec déclinaison des symptômes dans les toxidromes (Annexe 2).

La rédaction de ces documents synthétiques s'est inspirée d'un certain nombre de travaux récents (6-13).

\section{Recommandations pratiques}

Il nous a semblé important de formuler un certain nombre de recommandations pratiques. Nous nous sommes volontairement limités à l'essentiel.

Les recherches et dosages sériques ou plasmatiques par immunoanalyse doivent être réalisés après recueil du sang dans un tube sans gel séparateur.

En ce qui concerne l'alcoolémie médico-légale, deux techniques sont reconnues : la chromatographie en phase gazeuse (CPG) et la méthode de Cordebard. Tout résultat obtenu par une autre technique (enzymatique par exemple) entache la procédure de nullité et peut entraîner des poursuites contre l'expert ou contre le biologiste (14). Le dosage doit être réalisé sur sang total, l'alcoolémie sur sérum est en moyenne de $14 \%$ supérieure à celle sur sang total, et varie en fonction de l'hématocrite.

Pour la toxicologie analytique médico-légale, la mise en évidence du (des) toxique(s) et de son (ses) métabo- lite(s) est indispensable quelle que soit l'intoxication dans le cadre médico-légal. Lorsque les recherches et/ou les dosages sont réalisés par immunoanalyse : la confirmation de la nature du xénobiotique par une technique spécifique est obligatoire.

Quant à la toxicologie clinique médico-légale, quatre molécules doivent être recherchées dans le cadre du diagnostic de mort cérébrale : barbituriques, chloralose, éthanol, méprobamate.

\section{Le prélèvement conservatoire (Annexe 3)}

- Le prélèvement conservatoire : Pourquoi ?

La plupart des intoxications font l'objet de prélèvements biologiques en vue de déterminer la nature de l'intoxication, d'apprécier l'impact de celle-ci sur un certain nombre de grandes fonctions évaluées par le bilan biologique. Un nombre limité de molécules sont accessibles dans un délai compatible avec une décision thérapeutique (voir document biomarqueurs de toxicité et anomalies métaboliques dans les principales intoxications graves). Dans ces cas, le dosage sanguin permet de confirmer l'intoxication et de valider le recours à un traitement par un antidote lorsque celui-ci existe : $\mathrm{N}$ acétylcystéine dans l'intoxication au paracétamol, 4méthylpyrazole ou éthanol dans l'intoxication par l'éthylène glycol ou le méthanol, F-ab antidigoxine dans l'intoxication à la digoxine, par exemple. Dans d'autres cas, le délai nécessaire pour l'analyse du toxique est incompatible avec la nécessité d'un traitement médical par antidote : hydroxocobalamine dans l'intoxication cyanhydrique. Dans ces situations, divers marqueurs biologiques, disponibles rapidement sont extrêmement précieux, ils permettent une prise en charge médicale optimale de l'intoxiqué : $\mathrm{pH}$ et lactates dans l'intoxication par les cyanures ; numération, taux de prothrombine et complexes solubles dans l'intoxication par la colchicine par exemple. Enfin dans certaines intoxications, les marqueurs biologiques ne vont pas éclairer le clinicien sur la nature de l'intoxication, même s'ils sont utiles dans le traitement symptomatique, seule l'analyse toxicologique permettra de préciser la nature de l'agent causal. Ceci montre bien la nécessité de procéder dans un certain nombre de cas à des prélèvements conservatoires dès la prise en charge du malade. Il convient en effet de pratiquer sans délai ces prélèvements, en particulier sanguins, en raison de la demi-vie très brève de certains toxiques dans les milieux biologiques, celle-ci étant de 20 minutes pour la colchicine par exemple. Ces prélèvements seront ou ne seront pas analysés en fonction du contexte et de l'évolution de l'intoxication. Il convient également de rappeler qu'en cas d'admission tardive, le principe actif peut avoir été épuré du sang, seuls les métabolites pourront éventuellement être retrouvés : l'acide glycolique 
dans l'intoxication par l'éthylène glycol par exemple, ou l'acide formique dans l'intoxication par le méthanol. Le prélèvement médical conservatoire ne peut se substituer au prélèvement médico-légal qui fait quant à lui l'objet de recommandations spécifiques (15).

- Le prélèvement conservatoire : Quand prélever?

Un certain nombre de critères peuvent être retenus pour définir les conditions de réalisation de prélèvements à titre conservatoire :

- lorsque le résultat est utile pour le diagnostic de certitude de l'intoxication,

- lors de tout trouble ou altération de l'état général pouvant avoir une origine toxique,

- à chaque fois que le dosage présente un intérêt au plan de la prise en charge thérapeutique (antidotes...) et de la toxicocinétique,

- pour suivre l'évolution de l'intoxication et l'efficacité du traitement correcteur (toxiques cardiotropes, toxiques dialysables),

- si le caractère particulier de l'intoxication revêt une importance épidémiologique,

- dans tous les cas susceptibles d'avoir une incidence médico-légale,

- lors de toute pathologie récidivante inexpliquée.

- Le prélèvement conservatoire : Quels échantillons ?

Dès la prise en charge :

$10 \mathrm{ml}$ de sang sur héparinate de lithium SANS GEL SEPARATEUR (sauf en cas de suspicion ou d'intoxication avec un sel de lithium) :

- un tube de $5 \mathrm{ml}$ centrifugé décanté (plasma),

- un tube de $5 \mathrm{ml}$ non centrifugé (sang total)

Chez l'enfant selon les possibilités $2 \times 2,5$ ou $2 \times 3 \mathrm{ml}$. Le prélèvement peut être conservé à $+4^{\circ} \mathrm{C}$ (sauf cas particulier) si l'analyse est réalisée dans les 5 jours. En cas de dosage différé, il est souhaitable, après centrifugation, de congeler séparément le plasma et les globules, pour éviter la dégradation plus ou moins importante de certaines molécules, même à $+4^{\circ} \mathrm{C}$. La séparation du plasma et la conservation des globules est utile pour les toxiques à fixation érythrocytaire.

Lors du premier recueil d'urines :

Pour la recherche de stupéfiants, $10 \mathrm{ml}$ d'urines sur flacon neutre sans additif, ni conservateur, sont suffisants.

Pour les autres recherches, 20 à $30 \mathrm{ml}$ d'urines sur un flacon neutre sans additif ni conservateur, en notant la diurèse des 24 heures.

Elles peuvent être conservées à $+4^{\circ} \mathrm{C}$ ou congelées en fonction de la nature du toxique recherché et du délai séparant le recueil de l'analyse.
Plus tardivement et en fonction du contexte :

Une mèche de cheveux, ou en l'absence de cheveux, l'analyse peut être réalisée sur les poils axillaires ou pubiens.

- Le prélèvement conservatoire : Comment et à quel moment prélever?

Sang : le sang est prélevé en périphérique, et le plus précocement possible, à distance d'une perfusion (bras opposé) sur tube hépariné sans gel séparateur.

Urines : un échantillon des urines des 24 premières heures est conservé en notant la diurèse. Dans certains cas, il peut être utile de collecter séparément la première miction, ainsi après consommation d'héroïne, on ne retrouve le métabolite spécifique de l'héroïne (la 6monoacétylmorphine ou 6-MAM) que dans la miction qui suit la prise du stupéfiant en raison de sa demi-vie très brève : $2,4 \pm 0,8 \min (16)$.

Cheveux : 50 à 60 cheveux, soit le diamètre d'un crayon à papier, coupés au ras du cuir chevelu (et non arrachés) au niveau du vertex postérieur. Attacher solidement la mèche avant la coupe avec une petite ficelle près de la racine (ne pas utiliser d'adhésif). Il est important de pouvoir repérer la partie la plus récente des cheveux (côté racine), de la partie la plus ancienne (côté extrémité) en cas d'analyse segmentaire.

- Le prélèvement conservatoire : durée de recueil ?

Sang : la durée et l'intervalle des recueils sont fonction du délai entre la prise du toxique et la prise en charge du patient pour prendre en compte la vitesse d'absorption plus ou moins longue selon les médicaments ou la forme pharmaceutique en cause (forme retard par exemple), de la demi-vie du toxique, celle-ci étant très variable : 2 heures pour le paracétamol à dose thérapeutique, plusieurs semaines pour les anticoagulants oraux ou la chloroquine par exemple. Cette grande variabilité de la durée d'élimination du toxique peut conduire à réaliser de 2 à 3 prélèvements par 24 heures pendant une courte durée, à 1 prélèvement hebdomadaire pendant plusieurs semaines. Les dates et heures de prélèvement doivent être soigneusement mentionnées.

Urines : si le premier recueil d'urines apporte de nombreuses informations sur le «statut toxique» du malade, on admet que les urines sont une photographie de la consommation des xénobiotiques des 24 à 48 heures précédant le recueil. Ainsi la répétition des recueils ne présente pas d'intérêt.

Cheveux : la pousse des cheveux étant voisine de $1 \mathrm{~cm}$ par mois, chaque centimètre constitue le reflet d'exposition aux xénobiotiques d'un mois, il n'y a donc pas lieu d'envisager à priori d'autre prélèvement que celui effectué lors de l'admission. Il peut être intéressant 
dans certains cas de réaliser une analyse segmentaire de la mèche.

- Le prélèvement conservatoire : délai de conservation Celui-ci est fonction du diagnostic principal et/ou des diagnostics secondaires, ainsi que de l'évolution de l'état du malade. Après un délai de 8 jours, l'accord du prescripteur est demandé avant destruction, en raison de l'évolution parfois possible vers une affaire médicolégale.

\section{Conclusion}

Des praticiens de terrain connaissant bien la toxicologie : biologistes d'horizons variés, cliniciens réanimateurs ou de centre antipoison ont répondu aux orientations arrêtées par le conseil d'administration de la SFBC et mises en œuvre par le comité scientifique ( $\mathrm{Pr}$ P. Gambert, Dr J. Goudable), compte tenu de la nécessité d'une prise en charge globale centrée autour du malade. Les échanges entre les participants ont été très riches et le débat biologiste-clinicien permanent. Cette méthode de travail s'est révélée très fructueuse. Les documents synthétiques élaborés - et de ce fait incomplets - permettent malgré tout de livrer l'essentiel des informations utiles à la fois au clinicien et au biologiste généraliste. Lorsque ceux ci sont confrontés à une situation d'urgence, ils peuvent disposer des premières informations indispensables pour une prise en charge optimale des malades. Le groupe envisage une mise à jour régulière de ces documents en fonction des évolutions de la toxicologie analytique et de la prise en charge clinique des patients.

\section{Références}

1. Goullé J.P., Lacroix C. L'analyse toxicologique à l'aube du $3^{\text {ène }}$ millénaire. Ann. Biol. Clin. 2001 ; 59: 605-12.

2. Mégarbane B., Baud F. Intoxications aiguës médicamenteuses. EMC Toxicologie Pathologies Professionnelles $2002 ; 16-001-G-10: 1-31$.

3. Danel V. Actualité des traitements en toxicologie. 9ème Congrès Annuel de la Société Française de Toxicologie Analytique. La Clusaz, 12-16 mars 2001.

4. Office Français des drogues et toxicomanies : www.drogues.gouv.fr

5. Chamayou J. Les dangers du Haschich : les dernières découvertes scientifiques sur le cannabis. Thèse de Médecine. Rouen, 2002.

6. Malandain H., Cano Y. Rôle du laboratoire dans les intoxications aiguës par un alcool ou un glycol. Toxicorama $1999 ; 11$ (4) : 13-26.

7. Capolaghi B., Moulsma M., Houdret N., Baud F.J. Stratégies analytiques en toxicologie d'urgence. Ann. Toxicol. Anal. $2000 ; 12: 274-81$.

8. Capolaghi B., Charlier C., Feuillu A., Gruson A., Houdret N., Kergueris M.F., Lachatre G., Lacroix C., Lamiable D., Plomteux G. Toxiques et analyses biologiques en toxicologie hospitalière. Toxicorama $1998 ; 10$ (3) : 128-31.

9. Lheureux P., Maes V. Indications et interprétation des analyses toxicologiques. In Intoxications aiguës. Elsevier ed, Paris $1999:$ 61-77.

10. Lheureux P., Garbusinski J., Devuyst F., d'Eugenio S. Apport de la biologie et de la clinique dans l'évaluation des intoxications aiguës. Revue Française des Laboratoires 1999 ; 312 : 35-41.

11. Laforge M., Levillain P., Gourlain H., Buneaux F., Galliot-Guilley M. Analyses biologiques : critères indirects d'une intoxication aiguë. Rev. Fr. Laboratoires $1999 ; 312: 42-5$.

12. Alberta Clinical Practide, Guideline program : www.amda.ab.ca

13. Draft Guidelines of the Laboratory Support for Emergency Toxicology : www.nacb.org

14. Goullé J.P., Deveaux M. Expertises judiciaires en matière d'alcoolémie et de toxicologie. Ann. Biol. Clin., $2003 ; 15: 40-54$.

15. Pépin G., Deveaux M., Goullé J.P., Kintz P., Marquet P. Les prélèvements d'autopsie nécessaires à la bonne exécution des expertises toxicologiques. Toxicorama, 1998 ; $10(3): 110-9$.

16. Rentsch K.M., Kullak-Ulbick G.A., Reichel C., Meier P.J., Fattinger K. Arterial and venous pharmacokinetics of intravenous heroin in subjects who are addicted to narcotics. Clin. Pharmacol. Ther. 2001 ; 70 : 237-46. 
Annexe 1

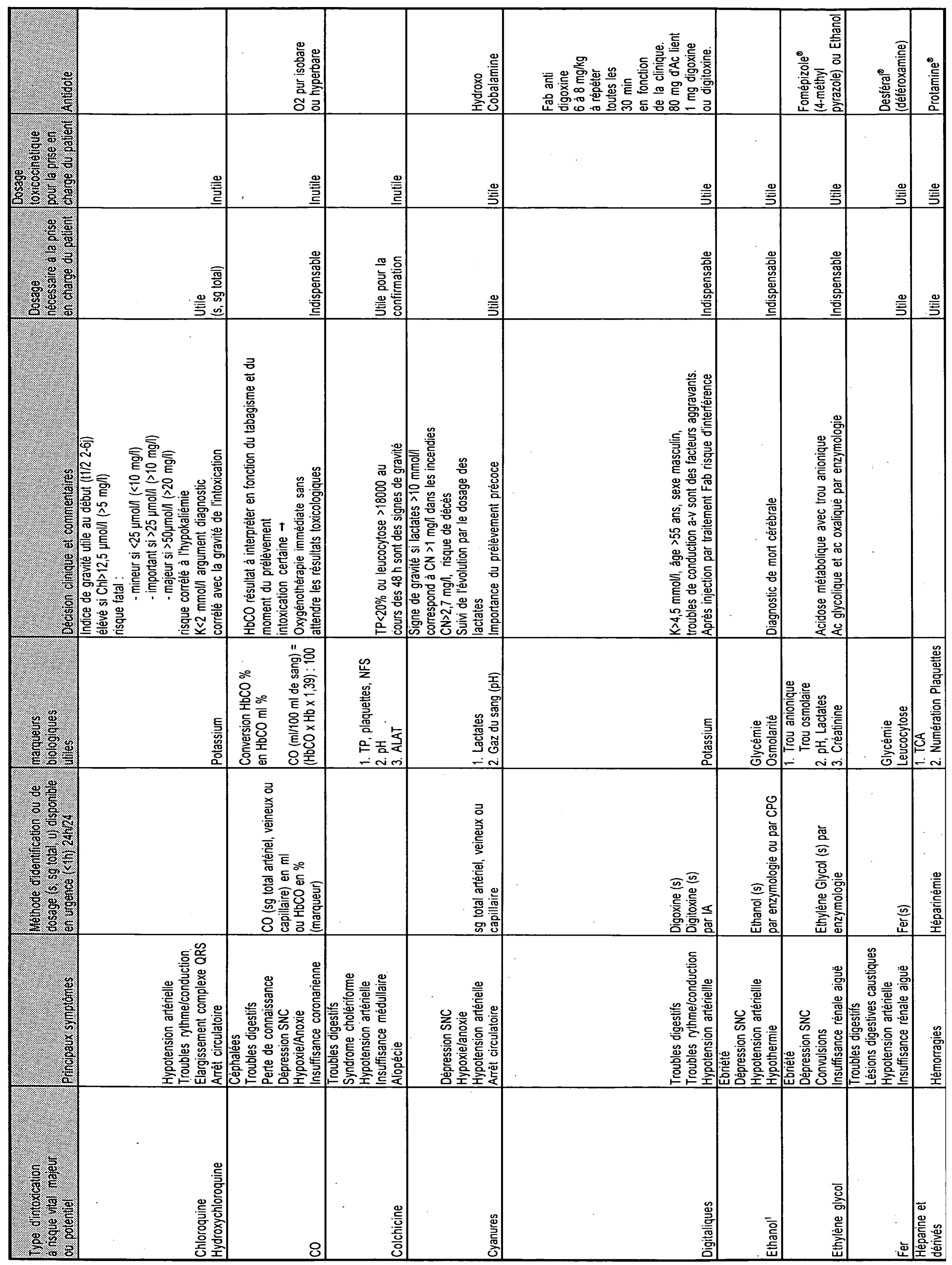




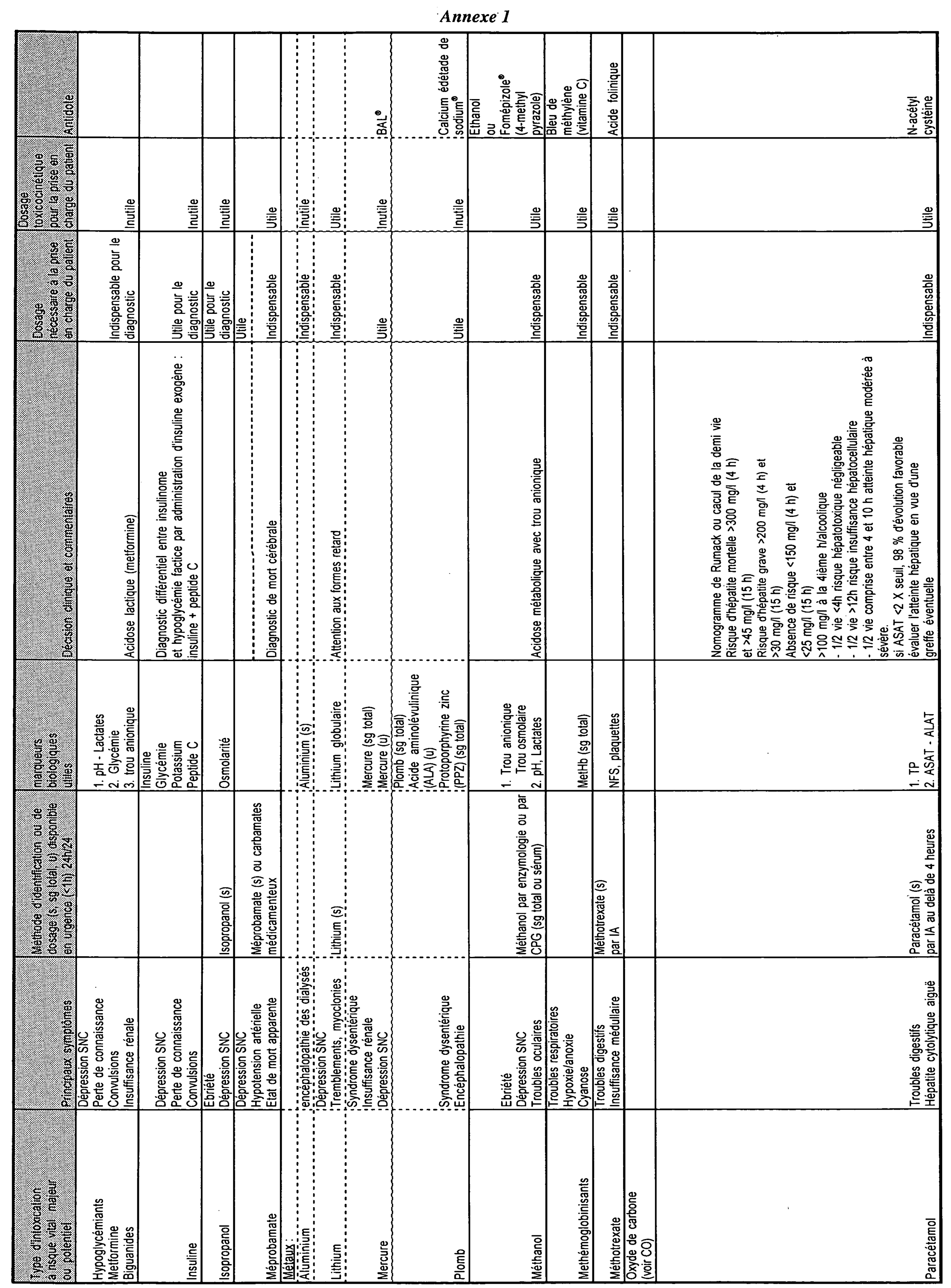


Annexe I

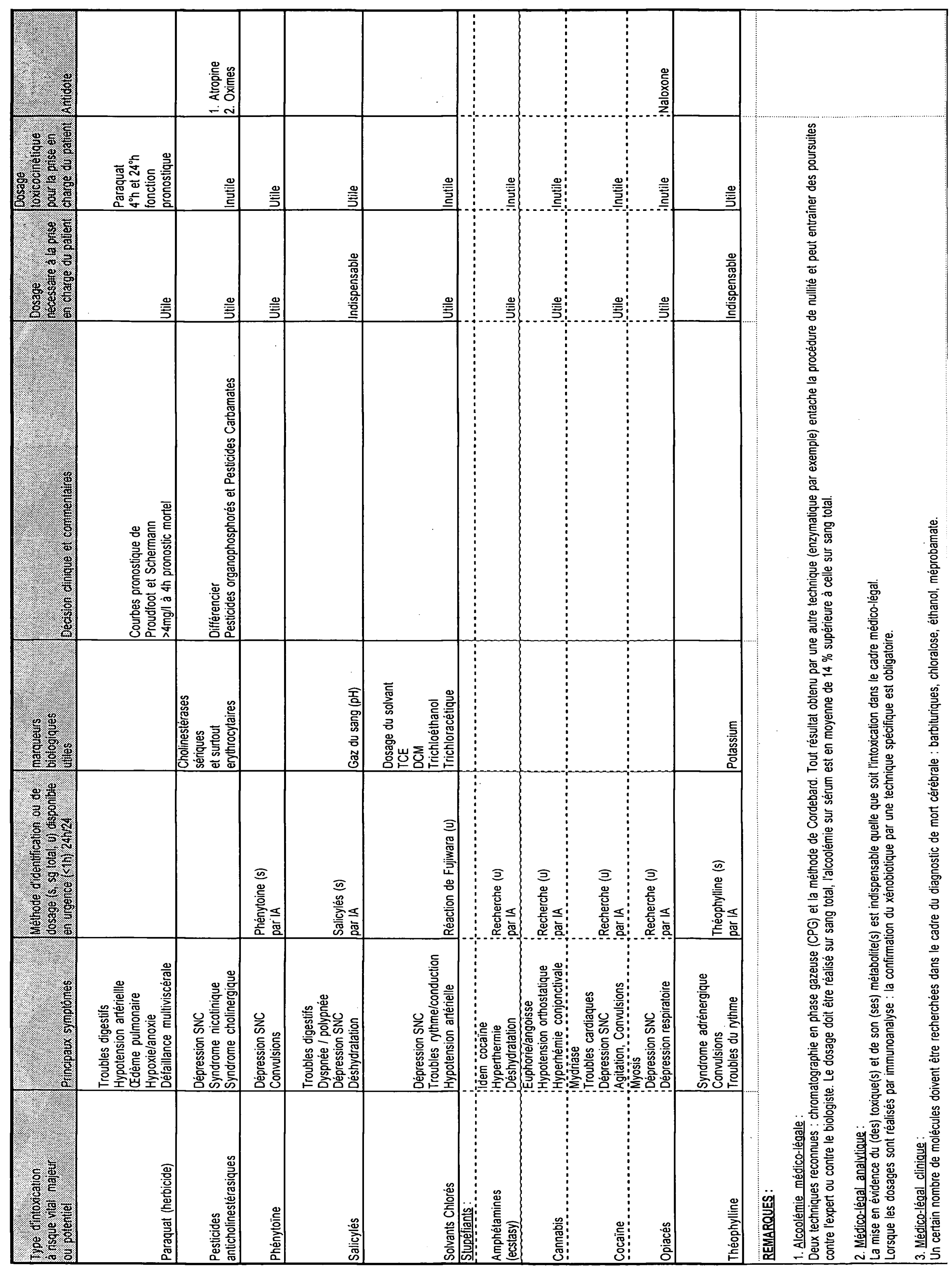


Annexe 2

\begin{tabular}{|c|c|}
\hline Symptômes & Produits \\
\hline Alopécie & Colchicine \\
\hline Arrêt circulatoire & $\begin{array}{l}\text { Chloroquine } \\
\text { Cyanures } \\
\text { Digitaliques }\end{array}$ \\
\hline Céphalées & $\mathrm{CO}$ \\
\hline Convulsions & $\begin{array}{l}\text { Acide valproïque } \\
\text { Antidépresseurs tricycliques } \\
\text { Antirouilles } \\
\text { Carbamazépine } \\
\text { Ethylène-glycol } \\
\text { Hypoglycémiants } \\
\text { Insuline } \\
\text { Phénytoïne } \\
\text { Théophylline }\end{array}$ \\
\hline Cyanose & Méthémoglobinisants \\
\hline Défaillance multiviscérale & Paraquat \\
\hline Dépression respiratoire / bradypnée & $\begin{array}{l}\text { Barbituriques } \\
\text { Bêta-bloqueurs } \\
\text { Benzodiazépines } \\
\text { Opiacés } \\
\end{array}$ \\
\hline Dépression SNC & $\begin{array}{l}\text { Acide valproïque } \\
\text { Antidépresseurs tricycliques } \\
\text { Barbituriques } \\
\text { Benzodiazépines } \\
\text { Bêta-bloqueurs } \\
\text { Brome et bromures } \\
\text { Carbamazépine } \\
\text { Chloralose } \\
\text { CO } \\
\text { Cyanures } \\
\text { Ethanol } \\
\text { Ethylène-glycol } \\
\text { Hypoglycémiants } \\
\text { Insuline } \\
\text { Isopropanol } \\
\text { Lithium } \\
\text { Méprobamate } \\
\text { Mercure } \\
\text { Méthanol } \\
\text { Pesticides anticholinestérasiques } \\
\text { Phénytoïne } \\
\text { Salicylés } \\
\text { Solvants chlorés } \\
\end{array}$ \\
\hline Déshydratation & Salicylés \\
\hline Dyspnée / Polypnée & Salicylés \\
\hline Ebriété & $\begin{array}{l}\text { Ethanol } \\
\text { Ethylène-glycol } \\
\text { Isopropanol } \\
\text { Méthanol }\end{array}$ \\
\hline Elargissement complexe QRS & $\begin{array}{l}\text { Antidépresseurs tricycliques } \\
\text { Chloroquine }\end{array}$ \\
\hline Encéphalopathie & Aluminium \\
\hline
\end{tabular}


Annexe 2

\begin{tabular}{|c|c|}
\hline & $\begin{array}{l}\text { Champignons } \\
\text { Plomb }\end{array}$ \\
\hline Etat de mort apparente & $\begin{array}{l}\text { Barbituriques } \\
\text { Chloralose } \\
\text { Ethanol } \\
\text { Méprobamate }\end{array}$ \\
\hline Hémorragies & $\begin{array}{l}\text { Anticoagulants AVK } \\
\text { Héparine et dérivés } \\
\text { Raticides AVK (chlorophacinone) }\end{array}$ \\
\hline Hépatite aiguë & $\begin{array}{l}\text { Champignons (amanite phalloïde) } \\
\text { Paracétamol } \\
\text { Solvants }\end{array}$ \\
\hline Hypotension artérielle & $\begin{array}{l}\text { Antidépresseurs tricycliques } \\
\text { Bêta-bloqueurs } \\
\text { Chloroquine } \\
\text { Colchicine } \\
\text { Cyanures } \\
\text { Digitaliques } \\
\text { Ethanol } \\
\text { Fer } \\
\text { Méprobamate } \\
\text { Paraquat } \\
\text { Solvants chlorés }\end{array}$ \\
\hline Hypothermie & $\begin{array}{l}\text { Barbituriques } \\
\text { Ethanol } \\
\text { Psychotropes }\end{array}$ \\
\hline Hypoxie / Anoxie & $\begin{array}{l}\text { CO } \\
\text { Cyanures } \\
\text { Méthémoglobinisants } \\
\text { Paraquat }\end{array}$ \\
\hline Insuffisance coronarienne & $\mathrm{CO}$ \\
\hline Insuffisance médullaire & $\begin{array}{l}\text { Colchicine } \\
\text { Méthotrexate }\end{array}$ \\
\hline Insuffisance rénale aiguë & $\begin{array}{l}\text { Ethylène-glycol } \\
\text { Fer } \\
\text { Hypoglycémiants } \\
\text { Mercure }\end{array}$ \\
\hline Lésions digestives caustiques & $\begin{array}{l}\text { Acides et bases } \\
\text { Antirouilles } \\
\text { Fer }\end{array}$ \\
\hline Mydriase & $\begin{array}{l}\text { Amphétamines } \\
\text { Cocaïne }\end{array}$ \\
\hline Myoclonies & \begin{tabular}{|l} 
Chloralose \\
Lithium
\end{tabular} \\
\hline Myosis & Opiacés \\
\hline CEdème pulmonaire & Paraquat \\
\hline Perte de connaissance & $\begin{array}{l}\text { CO } \\
\text { Hypoglycémiants } \\
\text { Insuline }\end{array}$ \\
\hline Syndrome adrénergique & Théophylline \\
\hline Syndrome cholériforme & $\begin{array}{l}\text { Champignons (phalloïde) } \\
\text { Colchicine }\end{array}$ \\
\hline Syndrome cholinergique & Pesticides anticholinestérasiques \\
\hline
\end{tabular}


Annexe 2

\begin{tabular}{|l|l|}
\hline Syndrome dysentérique & Mercure \\
Plomb
\end{tabular}




\section{Annexe 3}

\section{FICHE DE PRELEVEMENT CONSERVATOIRE}

NOM :

Prénom :

Date de Naissance :

Sexe :

Malade

Service ou UF :

Nom du médecin :

$\mathrm{N}^{\circ}$ de Téléphone :

Etiquettes d'identification

Service ou UF

Date et heure d'admission :

Renseignements cliniques
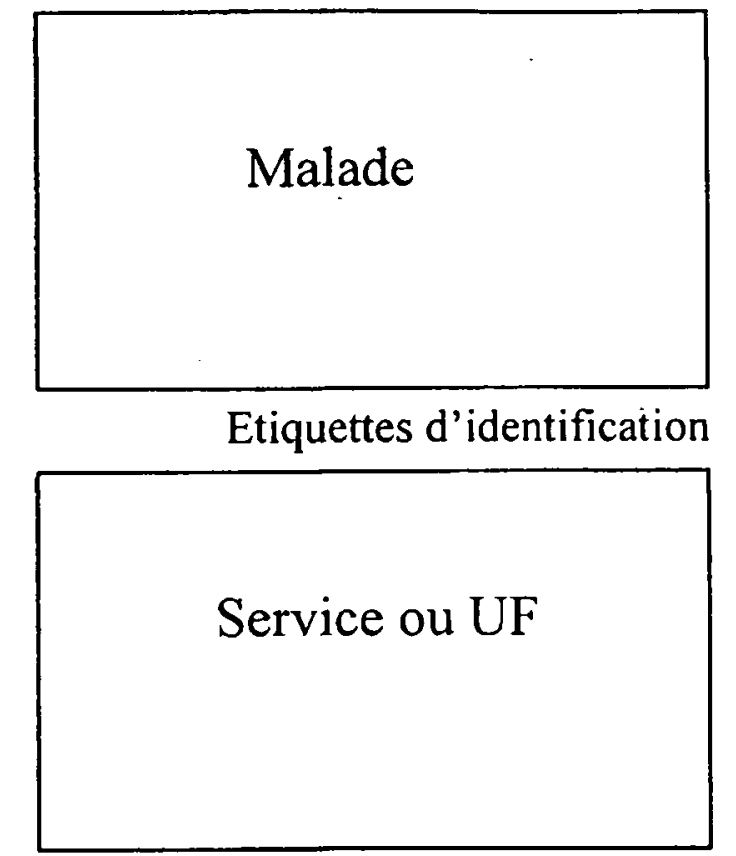

Résultat utile pour le diagnostic de certitude de l'intoxication

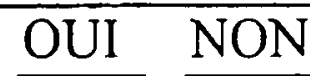

Intérêt toxicocinétique du dosage

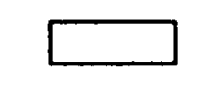

Importance épidémiologique du dosage

Observation susceptible d'avoir une conséquence médico-légale

Trouble ou altération de l'état général pouvant avoir une origine toxique

Pathologie récidivante inexpliquée

TOUTE CASE COCHEE « OUI » IMPOSE LE PRELEVEMENT

Date et heure de prélèvement (premier prélèvement)

- Sang :

- Urines :

- Cheveux :

Autres prélèvements (prélèvements suivants) :

Nature :

Fréquence : quotidienne-hebdomadaire - autre

Date de demande éventuelle d'analyse des prélèvements conservatoires :

Diagnostic principal :

Diagnostic(s) secondaire(s) :

Date de sortie du malade :

Durée de conservation des prélèvements en accord avec le prescripteur : 